\title{
Analysis on Curriculum Connection of Design Art in Universities against the Background of Intelligent Era
}

\author{
Hong Zhang \\ Beijing institute of technology, Zhuhai, Zhuhai 519088, China. \\ zhisme@126.com
}

\begin{abstract}
Curriculum cohesion in education field is a relatively neglected area. Because of this, the research of this problem has more practical significance. In the art of design field, the problem is more serious. On the one hand, the art of design has not to form a mature curriculum system, because Time of establishing is not long; on the other hand, many teachers come from art majors, they are more focused on perceptual thinking and lack of scientific research methods for theoretical research. Based on this, at the beginning of this article, it is pointed out some problems because of the neglect of course connection in the art of design field. In this paper, the author wants to explore the reasons for the improper connection between courses, and puts forward some corresponding solutions, by strengthening research on the curriculum system of design art, and to strengthen the understanding of "curriculum cohesion" among curriculum makers and curriculum practitioners; and to question The sequence of elements of curriculum cohesion ;etc.
\end{abstract}

Keywords: The art of design; curriculum system; curriculum cohesion; research.

\section{基于智能时代背景下高校设计艺术学科课程衔接窥探}

\section{张宏 \\ 北京理工大学珠海学院, 珠海 中国}

摘 要: 课程衔接在教育领域是相对被忽视的一个区域。也正因为此, 此问题的研究更具有实 际意义。在设计艺术学科, 课程衔接不顺畅的问题表现尤为突出。一方面是因为设计艺术学 科建立时间不久, 没有形成成熟的课程体系; 另一方面, 设计艺术学科由于很多教师都是出 身于美术专业, 更加偏重于感性思维, 对于理论研究缺乏科学的研究方法。基于此, 本文开 篇指出在设计艺术学科中, 由于忽视课程衔接所表现出的问题, 进而探究由于课程衔接不当 所产生的原因, 从而提出加强对设计艺术课程体系的研究; 加强课程制定者、课程执行者对 “课程衔接”的认识; 课程衔接的要素实施顺序要进一步商榷等等相应的解决办法。

关键词：设计艺术；课程体系；课程衔接；研究

\section{1. 前言}

随着《中国制造 2025》国家制造强国战略计划的提出，智能制造，成为新一轮科技革命的核 心, 社会的产业结构、人类的生活方式、以及社会对人才的需求都已发生了翻天覆地的变化。 而在智能时代背景下, 高校设计艺术学科课程衔接是否能顺应时代的发展, 应该要解决哪些 问题? 课程衔接如何能做到有针对性且紧密的联系, 需要我们认真加以研究和设计。

\section{2. 课程衔接不当所出现的问题}

在新开课程的授课过程中, 大部分教师都有过这样的经历, 对前一门课程已经学过的课程内 容进行询问时, 学生往往是一问三不知。然而, 在经过一番解释与引导过后, 学生似乎才能 记起之前已经学过。针对这种现象, 教师往往感到困惑, 是学生善忘还是之前的教师没有教 好课? 在这里, 我们要对一个概念进行理解和研究, 这就是 “课程衔接” 。作为课程组织重 
要的组成部分, 课程衔接也是课程设计的重要过程。它指的是不同的学习经验和课程内容进 行有效的连接, 从而使它们之间能因为产生累积而发挥出最大的功效, 以促成教学目标的达 成。课程如果没有合理的加以组织和衔接, 其知识就会变得支离破碎, 那么教育也就会变成 毫无意义的说教了。

\section{3. 课程衔接不当产生的原因}

3.1. 设计艺术课程体系的建立时间短

设计艺术学科课程从 1919 年德国包豪斯学校的建立到现今也不过短短的一百年时间, 整个的 课程体系并没有发展到完全成熟的阶段。尤其是对于中国设计教学而言, 从 20 世纪 80 年代, 国内开设设计艺术专业, 在课程设置上更多地是处于摸索阶段, 对设计艺术课程体系的研究 范围和深度都把握不够。

3. 2. 多数课程制定者、课程执行者对 “课程衔接” 概念认识不清楚

从课程的意义方面来说, 课程衔接的内容范畴较为广泛, 它既包括了课程目标、学科、教材、 计划, 也包括教师的教学方法、态度以及校园环境和学生的学习经验等等。在教学中往往出 现会学生无法适应前后不同教师所使用的不同的教学方法, 而导致其在课程衔接方面出现障 碍, 这属于教学方法的无法衔接。如若仅仅将教材或教科书等课程成品的衔接等同于课程衔 接, 这是非常狭险的观念。

课程制定者如果对课程衔接认识不清, 就会导致在排课过程中课程与课程之间的对接不够合 理。如某高校视觉传达设计专业, 就将基础课《平面构成》排在了《色彩构成》后面, 按正 常的排序应该《平面构成》在前, 《色彩构成》在后, 因为只有先解决平面造型问题, 才能 在后续的色彩设计中熟练地运用平面构成造型的法则。

此外, 课程制定者对课程衔接认识不清, 就不能很好地指导任课教师认识课程体系, 并导致 任课教师在课堂上未能积极引导学生对先后的课程内容进行合理衔接。如《色彩构成》是《设 计素描》和《平面构成》的后续课程, 然而在上《色彩构成》的过程中, 学生却只会专注于 对色彩知识点的学习, 在设计的图形中, 看不出对于构成知识的掌握和运用。之后在与学生 的交流中, 发现他们不懂平面构成的知识, 而是不懂得如何去运用。由此可见, 一门课程的 结束并不代表着对这门课程的学习和指导就结束了。在紧随其后的下一门课里, 任课教师就 很有必要在授课过程中有意对前续课程内容加以复述和引导, 启发学生将前后课程知识的学 习有效结合起来，从而达到事半功倍的效果。

3. 3. 课程制定者修订教学计划过程中，仅考虑显性课程设置

根据表现形态分类, 课程可以分为显性课程和隐性课程。显性课程是学校教学计划中明文规 定有计划、有组织, 由学术性内容的; 隐性课程则相对自由, 是处于教学计划之外的内容, 既可以是学术性的, 也可以是非学术性的。主要目的是为陶冶学生情感, 培养综合人文素养 而设定的。二者互为补充, 互相转化。它们都是建立课程衔接的重要内容。但在教学计划中, 课程体系仅关注了显性课程的构成。使得教师在授课中也只针对显性课程的内容进行阐述, 而对于教学计划外的内容如学生的学习态度、方法、接受程度等等极少关注, 忽视隐形课程 的作用, 由此对于课程开发、实施与效果之间容易产生脱节, 从而会使得显性课程的开发实 际应用价值不高。

3. 4. 设计艺术学科内基础课与专业课衔接不当

关于设计艺术教育中, 基础课程与专业课程的衔接一直是教学管理中颇为棘手的问题。其一, 任课教师之间缺乏有效的沟通。大学教师平时除了教学之外, 还有一定的科研任务需要完成, 因此上完课后, 也各自忙碌去了, 平时很少能有时间一起讨论教学及课程上的一些问题。其 二, 基础课程与专业课程一般是分由基础教研室和专业教研室分别管理, 两个部门之间平时 沟通较少。即基础是基础, 专业就是专业, 并不能成为一个有机的整体。其三, 对基础课程 教学的重视程度不够。从学生的角度看, 基础课程仅仅是打基础, 感觉是可有可无, 同时也 不知道怎样运用所学的基础知识为自己所学专业服务。因此, 基础课如何成为专业课的前提, 
专业课如何成为基础课的延伸与扩展, 两者之间如何衔接与过渡, 是设计艺术专业教学需要不 断研究和探讨的问题。

3. 5. 教师对所授课的学生群体了解不够深入

教师面对的学生是在不断变化的, 对于不同时代的学生, 其明显的时代特点需要教师变化教 学思路和方法。当前高校的学生, 大多是接近 “ 00 ” 甚至 “ 00 ” 后。出生在这个时代的孩子, 大多是个性比较强, 他们日常接触的事物相对丰富, 创新意识强, 对于一些新出现的知识讯 息的掌握程度要更为迅速, 对主流价值观很少盲目认同。此外, 这个时代的孩子由于物质条 件相对较好, 学习不是唯一出路, 他们中的大多数对学习没兴趣, 并且有强烈的叛逆意识。

\section{4. 加强课程衔接的对策}

4. 1. 加强对设计艺术课程体系的研究

设计艺术学科是极具人文性的一门学科, 其中课程内容的学习并不如数理化那般具有一定的 公式定理可循。其对人的影响是潜移默化和相对感性的。设计艺术学科又是极为年轻的一门 学科, 没有成熟的经验可以参考, 因此对于其中课程衔接的研究既要秉承严谨的科学理性精 神, 也要身体力行的参与实施与感受。应该在学习了解设计文化内涵的基础上, 深入分析课 程体系的设置。再通过结合当前的现状, 制定出合理有效的课程体系。课程衔接是一种课程 设计的理论, 有其认识论、教育观、课程观的理论基础, 不能仅仅被当做是安排教学计划的 方法，也不可仅流于技术层面的操作。

4. 2. 加强课程制定者、课程执行者对 “课程衔接” 的认识

当前教育界对 “课程衔接” 的概念不能形成统一的认识, 对于课程衔接的研究少之又少, 我 们可以参考的资料更是贵乏。但是我们应该明确的是课程衔接是非常值得研究的盲点, 其对 提升教学效果意义重大。其次, 我们也应明确, 课程衔接并不能孤立地去研究, 它其实是动 态的, 是一个连续的过程, 要瞻前顾后, 观左照右。课程衔接的研究要兼顾知识、学习者、 教师和教育环境等四个课程架构, 以免偏颇。

4. 3. 课程衔接的要素包括概念、主题、活动、内容、方式、能力等, 但是其实施顺序值得进 一步商榷

当然, 对于课程衔接模式的研究与选择应用, 教师要因时、因地制宜, 在课程实施过程中找 到最适合自身的, 相机使用。其实, 真正的课程衔接是发生在师生互动过程中。由于学生的 认知方式, 以及所接受的教材版本已相当多元, 因此教师在进行课程实施时, 应重视课程实 施的调适观和落实观, 从教学运行情境中落实课程衔接工作。

4. 4. 针对设计艺术学科特点, 加强基础课与专业课的衔接

设计艺术教学最主要的目的即启发和促成学生创造力和创新精神的形成, 并通过一定的形式 得以体现。这个目标在基础课与专业课的衔接过程中, 要达成共识。那么, 在课程的设置过 程中, 要加强基础和专业的融合。如可开设《造型基础》、《形式基础》、《思维训练》等 系列课程, 将基础训练同专业设计有机地联系起来。在《造型基础》中强调以丰富灵活的训 练课题, 启发和引导学生创造性地理解艺术与设计的关系、绘画基础与设计基础的关系。课 程训练内容主要以素描为基本手段, 联系形式构成语言以及材料实验的内容。对现实形态、

自然形态、人工形态等进行写生分析变体训练, 从而完成从现象到本质、从物象到心像、从 具象到抽象、从感性到理性的过渡。

在包豪斯设计学院的教学体系中, 是通过广泛地开设 “观察”、“分析”、“研究” 等课程, 启发学生对造型、色彩、结构的分析和表现能力来达到培养学生综合创作能力的。在约翰. 伊顿的基础课程训练中, 强调形式和色彩的客观分析, 注重点、线、面的关系。通过实践, 使 学生了解如何客观地分析两度空间的构成, 并进而推广到三度空间的构成上。因此, 《形式基 础》课程的教学目标是要让学生掌握形式的基本原理和法则, 学会在自然与现实生活中观察 和发现形式, 最终灵活地运用和创造形式。课程包含了二维平面形式、色彩形式和三维立体 形式, 进而扩展到对四维形式的关注。从自然、艺术、设计方面阐述形式的作用, 力图从来 
源于自然的形式入手阐释形式在自然中的功能作用, 以及自然的形式对仿生学设计的启发, 从来源于艺术的形式阐述形式对于精神表现的作用, 以及形式在艺术中的独特呈现, 从来源 于设计的形式证明形式的设计效用, 即形式的功能与审美的双重作用。《思维训练》课程的 训练目的是锻炼人, 是对于自我的发掘和完善。课程将艺术中的视觉表现与形象思维、设计 中的分析与计划相结合, 通过训练拓展学生的思维和想象力, 提高他们创造的主动性, 为学 生奠定设计思维的基础，提升设计作品的文化内涵和审美感。

加强基础课与专业课的衔接, 亦可打通基础与专业的界限, 削弱 “基础”、“专业” 的概念。 将大学四年的课程相互之间进行打通, 以 “培养人才” 作为办学的依托点, 而不是通过分级 的课程。在整个教学过程中, 引入课题, 以课题促进教学, 培养学生自我发展意识与能力。 一门课程可以由几位老师共同指导, 在课程开始, 教师先行布置课题, 提出课题基本要求, 引入必要的设计实例作参考。学生根据课题要求, 自行寻找完成课题的途径和方法, 可以根 据不同的问题求教于不同的教师。这种教学模式, 由于没有固定的思维模式限制, 学生更能 够积极有效地主动进行探索式学习, 从而也能够更自由地发挥个性, 建立起自主学习的乐趣。 另外, 由于一门课程需有多名教师共同授课, 使得授课教师之间必然就教学中出现的问题要 多做交流和沟通, 从而也就更好地保证了课程质量。总之, 基础课与专业课进行合理有效的 衔接要做到既不重复又不断层。另外, 在课程的设计与上课时间的安排上都应做到对比与统 一的原则。

4. 5. 成功的课程衔接需要学科专家、教育学者、教师和学生等多方对话与协商 缺少任何一方的积极参与, 其结果都是不理想的。因此要经常性的创造可以产生对话的机会, 亦可通过合适的途径, 进行沟通。如可以将设计艺术学科专家、教育学者的emai1公布于普通 教师, 当教师遇到问题时, 可以通过电子邮件的形式寻求帮助; 或者学科专家和教育学者要 多下高校课堂, 与教师学生以座谈会的形式解决问题; 同时, 教师在课堂上要重视学生学习 的过程, 主动与学生进行教学方面的沟通和交流。总之, 设计艺术学科要真正将课程衔接落 到实处, 就一定要重视人与人之间的相互沟通与互动, 以建立共识。

\section{5. 小结}

对于课程衔接, 我们不能仅仅将其当做是一个名词概念。对待课程衔接, 要抱着一种动态的 课程理念去审视, 绝不能将其当做是教学过程中两个教学内容的过渡。对于设计艺术课程衔 接, 我们更应该考虑到设计艺术的学科特点, 用一种包容的, 非概念性的方式去解读、分析、 实践, 在立足实践、立足于学生终身发展、和谐发展的基础上, 充分调动多方的积极性, 合 理探讨, 力求使设计艺术学科的课程衔接既具有自身的独立性, 又具有相对的合理性。

\section{References}

[1]. (us) Tom Russ. Sustainability and design ethics [M]. Chongqing: Chongqing university press, 2016.11.

[2]. Klaus Lehman. Design of education [M]. Nanjing: Jiangsu phoenix art publishing house, 2016.10.

[3]. Zhou shay. A collection of basic teaching works of the school of design of central academy of fine arts [M]. Beijing: China youth press, 2011.3.

[4]. Wang cueing/Zheng meaning. Two-dimensional design basis [M]. Shanghai: Shanghai people's fine arts publishing house, 2016.1.

[5]. Hu changing. Curriculum cohesion: meaning analysis, theoretical basis and major problems [J]. Wuhan business Service College. 
[6]. Zhang Hong. A number of reflections on the structural reform of college design art courses [J]. Design.2016.10. 